\title{
Food cravings, aversions and pica among pregnant women in Dar es Salaam, Tanzania
}

\author{
C.N.M. NYARUHUCHA \\ Sokoine University of Agriculture, Department of Food Science and Technology, \\ P.O .Box 3006, Morogoro, Tanzania \\ E-mail:nyaruhu@suanet.ac.tz
}

\begin{abstract}
Food cravings, aversions and pica are common during pregnancy and may have a significant input on pregnancy progress and outcome. A study was carried out to determine the frequency and duration of pronounced dietary cravings, aversions and pica during pregnancy among 204 pregnant and lactating women attending two health facilities in Dar es Salaam City, Tanzania. Nausea and vomiting were reported by $82.8 \%$ of all women of which $43.2 \%$ experienced severe nausea alone, 9.5\% severe vomiting alone and $35.5 \%$ experienced severe vomiting and nausea. Mild cases of each of the symptoms either occurring alone or both of them occurring together were also reported. Both behaviours were observed more in $<3$ months of pregnancy. The proportions of women with dietary cravings, aversions, and pica were $73.5 \%, 70.1 \%$ and $63.7 \%$ of all women respectively. More women $(70.1 \%)$ experienced both food cravings and aversions than either symptom alone. Foods craved most were meat $(23.3 \%)$, mangoes $(22.7 \%)$, yoghurt $(20.0 \%)$ oranges $(20.0 \%)$, plantain $(15.3 \%)$ and soft drinks (13.3\%). Foods avoided most were rice (36.4\%), meat (36.4\%) and fish (30.8\%). Eggs, beans, tea and stiff porridge were also avoided. Reasons given for avoiding foods were unpleasant smell/taste $(10.3 \%)$, to reduce nausea (11.8\%), no particular reason (58.3\%) and dislike by foetus (belief) (3.9\%). Pica was experienced by $63.7 \% \%$ of the women and soil, ice and ash were the most commonly non-food substances eaten. The frequency of nausea and vomiting was highest in the early months of pregnancy and most women experienced the symptoms during morning hours. Craving in most women was more intense in the first trimesters. Most women craved for meat and sour and savoury foods, and avoided rice, meat and fish. Soil consumption was the pica observed in most women. Since aversions and cravings are closely linked to dietary intake of pregnant woman understanding these behaviours is important in addressing maternal nutrition.
\end{abstract}

Key words: food, craving, aversion, pica, pregnancy, women, Tanzania

\section{Introduction}

Pregnancy is often accompanied by a variety of nutritionally linked problems with symptoms that are sometimes very unpleasant and difficult to tolerate (Doerr, 2001). Cravings and aversions, which refer to a strong desire and strong dislike respectively for certain food, are common during pregnancy such as nausea and vomiting (Walker et al., 1985; Caplan, 2001). These complications may cause not only discomfort during pregnancy but also interfere with the dietary intake of the pregnant woman and sometimes causing serious problems (Caplan, 2001). One of the most prevalent examples of the absence of comfort during pregnancy is the occurrence of morning sickness; which occurs often in women in their early stages of pregnancy. Morning sickness is commonly linked with the food aversions, cravings, nausea, and vomiting that are often associated with pregnancy. Up to twothirds of all pregnant women may suffer from any combination of these symptoms (Walker et al., 1985).)

Some theories of the reasons for morning sickness have been developed, yet no specific cause has been identified (Caplan, 2001). There is similar dearth of information on the consumption of non-nutritive substances (pica) during pregnancy (Coronios-vargas et al., 1991). Human pica refers to the compulsion for persistent ingestion of unsuitable substances (e.g. soil, clay, chalk, ice, etc.) having little or no nutritional value (Darkish et al., 1982). Such non-food substances include soil, clay, ice, burnt matches, soot, charcoal, cigarette ashes, and baking soda (Walker et al., 1985). The etiology of pica is poorly understood although several proposals have been made. One theory suggests that the ingestion of non-food substances relieves nausea and vomiting. Another theory suggests that the deficiency of essential nutrient such as calcium or iron results in the eating of non-food substances that contain these nutrients (Tierson, 1997). Displacement effect of non-food substances could result in reduced intake of nutritious foods leading to inadequate dietary intakes of essential nutrients. Some pica substances may contain toxic compounds or quantities of nutrients not tolerated in disease states, while some pica substances interfere with the absorption of certain mineral elements such as iron (Crosby, 1982).

There is lack of information on patterns, duration, frequency and causes of cravings and aversions, pica, nausea and vomiting in 
pregnant women in Tanzania. This study was therefore carried out to generate information on abnormal food habits during pregnancy in Tanzania by using Dar es Salaam City as a case study. The objective of this study was to assess the frequency of pronounced dietary cravings and aversions and to identify most common foods that pregnant women crave or dislike during pregnancy. Such information would assist nutrition/health workers to help pregnant women make a better choice of food during pregnancy.

\section{Materials and Methods}

\section{Study area and subjects}

The study was conducted in Dar es Salaam City in Tanzania. The study enrolled all apparently healthy pregnant women and lactating mothers (who had children $\leq 4$ months) attending Reproductive and Child Health clinics between June 2005 and February 2006. Two RCH clinics at Mwananyamala Hospital and Magomeni Health Centre were selected for the study.

\section{Data collection}

Questionnaires were designed to gather information on women's experiences on dietary modifications (aversion and craving during pregnancy), vomiting and nausea. On food craving and aversion, subjects were asked to write (in order of preference) foods, which were craved for and/or avoided. They were also asked if they did feel urge to eat any substance not normally eaten including, ash, sand, chalk, starch. With regard to nausea and vomiting subjects were asked if during their last pregnancy they felt mild or severe nauseous during the first 3 months, or throughout pregnancy. Mild nausea or vomiting implies that such occurred either only mildly or only occasionally, and was not particularly distressing; severe nausea or vomiting implies that it occurred severely almost every day and occasionally throughout the day. Women who had more than one child were asked about their previous experiences of nausea and vomiting.

\section{Data analysis}

Data were processed and analyzed using Statistical Package for Social Scientist (SPSS) software version 9.0 for windows (Norusis and SPSS, 1995). Descriptive statistics were used to get the percentages and frequencies of variables used in this study.

\section{Results}

\section{Subjects' characteristics}

A total of 204 women participated in the study, of which $121(59.3 \%)$ were $\leq 25$ years of age, $30 \%$ were between $26-30$ years of age and only $19 \%$ were over 30 years of age. Of the subjects, $156(76.5 \%)$ were lactating while the rest were pregnant. Ages of pregnancies ranged from first to third trimesters and the number of pregnancy experiences ranged from one to four.

\section{Cravings and aversions}

The proportion of women who reported pronounced cravings and aversions was $75.5 \%$. Out of these, $70.1 \%$ experienced both cravings and aversions of certain foods, $3.4 \%$ experienced cravings alone and $2.0 \%$ aversions alone. Most $(72.1 \%)$ women experienced food cravings and aversions rather than craving alone $(3.4 \%)$ or aversion $(2.0 \%)$ alone. Food cravings were reported in $73.5 \%$; out of these, $34.3 \%$ craved one food while $39.2 \%$ craved more than one food. Intensity of cravings was reported to be highest $(43.6 \%)$ in the first trimester than in the second $(21.6 \%)$ and third trimesters (5.4\%). Only a small proportion (2.9\%) of women reported to maintain their cravings throughout pregnancy period although with less intensity. Food aversion was reported in $70.1 \%$; out of these $30.9 \%$ disliked one food while $39.2 \%$ disliked more than one food. 
Table 1: Proportion of pregnant women with craving for specific foods or food categories

\begin{tabular}{llll}
\hline Group of food & Food & No. of respondents & Percent \\
\hline Specific food & Meat & 35 & 14.0 \\
Mangoes & 34 & 13.6 \\
Yoghurt & 30 & 12.0 \\
Oranges & 30 & 12.0 \\
Plantain & 23 & 9.2 \\
Soft drinks & 20 & 8.0 \\
Amaranths & 17 & 6.8 \\
& Sweet potato leaves & 15 & 6.0 \\
& Lady's finger & 11 & 4.4 \\
& Fried potatoes & 11 & 4.4 \\
Sweets & 9 & 3.6 \\
Eggs & 5 & 2.0 \\
Cowpeas & 4 & 1.6 \\
& Stiff porridge & 4 & 1.6 \\
& Milk & 2 & 0.8 \\
& Fruits & 87 & 34.8 \\
& Vegetables & 58 & 23.2 \\
Meat and fish & 35 & 14.0 \\
& Dairy & 32 & 12.8
\end{tabular}

The most widely craved foods were meat, mangoes, yoghurt, oranges, plantain and soft drinks (Table 1). When craved foods were aggregated, fruits were craved by the largest proportion of women $(34.8 \%)$ followed by vegetables $(23.2 \%)$.

The most commonly avoided foods were rice, meat and fish, which were avoided by $36.4 \%, 36.4 \%$ and $30.8 \% \%$ of the women, respectively. When the foods were divided into categories, meat and fish were disliked by the largest proportion of women $(67.0 \%)$ followed by grains and grain products $(47.7 \%)$. Other categories were avoided by a smaller proportion of women (Table 2).
The majority of the women $(58.3 \%)$ did not have reasons for their food aversions. However, $10.3 \%$ of the women insisted that aversions were caused by changes in the taste and/or smell of the food during pregnancy, $11.8 \%$ believed that aversions of certain foods help to overcome the symptoms of nausea and vomiting. Some women $(58.3 \%$ had no particular reason for avoiding foods while a few (3.9\%) believed that aversions were caused by "a dislike" of the food by the foetus.

\section{Pica}

A total of 12 women reported to have experience pica during pregnancy. Pica was reported by

Table 2: The number (\%) of respondents and aversion for specific foods or food categories

\begin{tabular}{llll}
\hline Group of food & Food & No. respondent & Percent \\
\hline Specific food & Rice & 52 & 36.4 \\
& Meat & 52 & 36.4 \\
& Fish & 44 & 30.8 \\
& Eggs & 15 & 10.5 \\
& Beans & 14 & 9.8 \\
& Tea & 13 & 9.1 \\
& Stiff porridge & 12 & 8.4 \\
& Sweet potato leaves & 7 & 4.9 \\
& Meat and fish & 96 & 67.0 \\
& Grain/grain products & 64 & 47.7 \\
& Meat alternatives & 15 & 10.4 \\
& Legumes & 14 & 9.8 \\
& Vegetables & 13 & 9.1 \\
& Beverages & 13 & 9.1 \\
& Dairy & 5 & 3.5 \\
\hline
\end{tabular}


$63.7 \%$ of the women. The consumption of soil commonly called Udongo wa Pemba (soil from Pemba Islands) was reported among $60.0 \%$ of affected women followed by consumption of ice $(16.1 \%)$, ash (15.4\%) and other substances such as charcoal, clay, bar soaps and dust $(<3 \%)$.

\section{Nausea and vomiting}

A total of 169 (82.8\%) women had experienced nausea and vomiting for different durations during their pregnancy. Out of the women affected $43.2 \%$ experienced severe nausea alone, $9.5 \%$ had severe vomiting without nausea and $35.5 \%$ experienced severe nausea and vomiting. Mild cases of each of the symptoms either occurring alone or occurring together were also reported (Table 3). Among the affected women, $59.3 \%$ stated that the symptoms were most frequent between $12^{\text {th }}$ and $16^{\text {th }}$ week of pregnancy.

\section{Discussion}

The relatively high prevalence of cravings observed in the present study was similar to that reported by other researchers, which ranged from 67 to $84 \%$ (Crosby, 1982; Walker et al., 1985; Demissie et al., 1995). Women who experience food aversions also crave for at least one or more foods not only to compensate but also to diversify their diets and to consume higher-quality foods. Milk and milk products are an important part of the diet because of the excellent balance of nutrients, and particularly because of the calcium and riboflavin content. In addition, they provide some of practically all other essential nutrients in well-balanced amounts and in easily assimilated forms. The avoidance of such foods would therefore reduce the quality of foods eaten by pregnant women.

Table 3: Number and percent of respondents who experienced nausea and vomiting during pregnancy

\begin{tabular}{|c|c|c|c|}
\hline Type of experience & $\begin{array}{l}\begin{array}{l}\text { Duration of experience } \\
\text { (months) }\end{array} \\
\end{array}$ & No. respondents & $\%$ \\
\hline \multirow[t]{2}{*}{ Severe nausea } & $0-3$ & 63 & 37.3 \\
\hline & $0-9$ & 10 & 5.9 \\
\hline \multirow[t]{2}{*}{ Mild nausea } & $0-3$ & 10 & 5.9 \\
\hline & $0-9$ & 2 & 1.2 \\
\hline \multirow[t]{2}{*}{ Severe vomiting without nausea } & $0-3$ & 13 & 7.7 \\
\hline & $0-9$ & 3 & 1.8 \\
\hline \multirow[t]{2}{*}{ Mild vomiting without nausea } & $0-3$ & 3 & 1.8 \\
\hline & $0-9$ & 1 & 0.6 \\
\hline \multirow[t]{2}{*}{ Severe vomiting and nausea } & $0-3$ & 45 & 26.6 \\
\hline & $0-9$ & 15 & 8.9 \\
\hline \multirow[t]{2}{*}{ Mild vomiting and nausea } & $0-3$ & 4 & 1.1 \\
\hline & $0-9$ & 0 & 0 \\
\hline
\end{tabular}

Twenty-eight percent of the women reported that there was no difference in terms of frequency and severity of nausea and vomiting between pregnancies. However, $12.3 \%$ insisted that the first pregnancy was associated with greater severity of sickness and $6.4 \%$ reported to have experienced greater severity of the symptoms during the second or later pregnancy. Of the women affected, $40.2 \%$ reported to experience nausea/vomiting most often in the morning, $16.7 \%$ in the afternoon, $8.8 \%$ throughout the day, $5.4 \%$ at night and $1.5 \%$ during the evening hours. Of the women who experienced the nausea during the afternoon, it was most often after a meal. However, this also depended on the kind of food taken. Women avoided certain foods just to overcome the occurrence of nausea/vomiting and craved for certain foods that seemed not to trigger their stomach upsets.
This would therefore necessitate eating of other high protein quality foods to meet their nutrient requirements. In study, this was compensated for by high cravings for meat and fish, other foods with high protein quality. The avoidance of cereals observed in this study supports the observation that pregnant women tend to avoid foods commonly eaten to avoid monotony and to diversify the foods they consume (Demissie et al. 1995).

The reasons for the changes in eating habit were not clear. Some of the reasons given included the beliefs that cravings or aversions is caused by "a dislike" of food by the foetus, unpleasant smell, vomiting and heart burn. In this study, women also reported drastic changes in their response to smell and taste foods to be associated with food cravings and 
aversions. These changes, however, are likely to be associated with hormonal hyperactivity during the first trimester. It has been observed that there is an increase in saliva, which causes pregnant woman's mouth to water more than usual (Demissie et al., 1995). The saliva that reflects the hormonal changes occurring in the body may also produce a slight metallic taste in the mouth (Coronios-vargas et al., 1991). According to some observations, cravings and aversions are likely to be due to a dulling of taste sense during pregnancy (Hytten \& Letch, 1971).

The proportion of pica consumption is high among women in Dar es Salaam. The eating of soil (geophagia) was reported by most women and others even insisted that soil consumption during pregnancy is normal and essential thing. In case of soil consumption, while the practice may contribute significant amounts of calcium and trace elements, under certain conditions it may exacerbate existing iron deficiency anaemia (Tierson, 1997). The extent to which iron deficiency causes geophagia or geophagia promotes iron deficiency is not clear but some evidences from South Africa strongly favour the former view (Coltman, 1969; Crosby, 1982). In contrary, Sayers et al. (1974) reported that the eating of local earth does not have a deleterious effect on iron nutrition in the vast majority of cases. Some women in this study commented that eating of soil/clay is a custom stemming from deeply imbedded cultural traditions and attitudes.

Nausea and vomiting were common among pregnant women in Dar es Salaam. The frequency of nausea and vomiting is highest in the early months of pregnancy and most women experience the symptoms during morning hours. Craving in most women was more intense in the first trimesters. Most women craved for meat and sour and savoury foods, and would avoid rice, meat and fish. Soil consumption was the most common pica observed in most women and its consumption was considered a common culture among pregnant women. Similar observations have been by other researchers in Africa (Walker et al., 1985). However, the severity of sickness differs from one pregnancy to another (Soules et al., 1980). A woman could be very sick in one pregnancy and yet much less affected or even not affected at all in a previous or in a subsequent pregnancy. In this study only a few women reported to experience the severity of symptoms with the second or later pregnancy. Most women in this study experienced nausea/vomiting during the morning hours, a phenomenon referred to as "morning sickness". Unlike in our study, Caplan (2001) noted nausea and vomiting among pregnant women is experienced not only during the morning hours but also during all hours of the day equally. Maternal dietary cravings and aversions during pregnancy tend to be closely related to vomiting and nausea (Tierson, 2003).

In conclusion, since aversions and cravings are closely linked to dietary intake of pregnant woman understanding these behaviours is important in addressing the issue of maternal nutrition in a pregnant woman. Unhealthy cravings for non-food items should be discouraged as there is no known nutritional benefit of such habit and can lead to intestinal problems like abdominal pain and infection or any other health concerns. Excessive vomiting may lead to an inadequate intake of protein and energy and the loss of essential vitamins, minerals and electrolytes; it is important that a pregnant woman eats well when she is not feeling nauseous. Given the importance of nutritional value and composition of foods consumed during pregnancy health workers/ nutritionists may use these findings to provide appropriatenutrition counselling and education to guide women make a wise choice of foods in order to improve their health and nutritional status during pregnancy.

\section{Acknowledgements}

I am grateful to Sokoine University of Agriculture for financial support, and the administrators of Reproductive and Child Health clinics at Mwananyamala Hospital and Magomeni Health Centre in Dar es Salaam for permitting data collection.

Received 18 November 2008

Revised 17 December 2008

Accepted 17 December 2008

\section{References}

Caplan, C.D. (2001) Evolutionary causes and importance of pregnancy sickness: (http://www.geogle.com/ derekeqplan.htm) site visited on 26/08/2006.

Coltman, C.A.J.(1969) Papophagia and iron lack. 
Journal of American Medical Association 207, 513-515.

Coronios-vargas, M., Toma, R.B., Tuveson, R.V. \& Schutz, I.M. (1991) Cultural influences on food cravings and aversions during pregnancy. Ecology of Food and Nutrition 27, 43-49.

Crosby, W.H. (1982) Clay ingestion and iron deficiency. Annals of Internal Medicine 97, 45-67.

Darkish, O.A., Amine, E.K. \& Abdullah, S.M. (1982) Food habits during pregnancy and lactation in Iraq. Food and Nutrition Bulletin 4, 14-16.

Demissie, T., Mirroki, N.M. \& Wambui, K. (1995) Food cravings and aversions during pregnancy: prevalence and significance for maternal nutrition in Ethiopia.:(http://www.unu.edu/ unupress/food/V191e/eho5.htm). Accessed on 20/03/2006.

Doerr, M. (2001) Morning sickness, nausea and vomiting during pregnancy: http:// www.storknet.com/complications/ morningsickness/nausea.htm). Accessed on 26/08/2006.

Hytten, F.E. \& Letch, I. (1971) Physiology of Human Pregnancy. $2^{\text {nd }}$ Edition, Blackwell
Scientific Publishing, London. Pp. 310329,

Norusis, M.J. \& SPSS, Inc. (1995) Data analysis for SPSS/PC+TM. Version 9.0. Chicago, Illinois. Pp 24-47.

Sayers, G., Lipschitz, A.D., Sayes, M. \& Charton, R.M. (1974) Relationship between pica and iron nutrition in Johannesburg black adults. South African Medical Association 48, 1655-1660.

Soules, M.R., Hughes, C.L., Garcia, J.A., Livengood, C.H. \& Alexander, E. (1980) Nausea and vomiting in pregnancy: role of human chorionic gonadotrophin and 17-hydraxyprogestorone. Obstetrics and Gynaecology 55, 596-700.

Tierson, F.D. (1997) Pregnancy and nausea and vomiting. Encyclopedia of Human Biology. $2^{\text {nd }}$ Ed. Academic Press, Amsterdam.

Tierson, F.D. (2003) Nausea and vomiting of pregnancy and its association with pregnancy outcome. (http//www.nup. volumes-org/p1-8.htm). Accessed on 26/08/2006.

Walker, A.R.P., Walker, J.J., Verardi, M. \& Walker, C. (1985) Nausea and vomiting, cravings and aversions during pregnancy in South African women. British Journal of Obstetrics and Gynaecology 92,484-489. 